\title{
Effects of Magnetohydrodynamics Matter Density Fluctuations on the Solar Neutrino Resonant Spin-Flavor Precession
}

\author{
N. Reggiani ${ }^{a *}$, M.M. Guzzo ${ }^{b \dagger}$ J.H. Colonia ${ }^{b \ddagger}$, and P.C. de Holanda ${ }^{b, c \S}$ \\ ${ }^{a}$ Instituto de Ciências Exatas \\ Pontifícia Universidade Católica de Campinas \\ 13020-970 Campinas, SP, Brasil \\ ${ }^{b}$ Instituto de Física Gleb Wataghin \\ Universidade Estadual de Campinas - UNICAMP \\ 13083-970 Campinas, São Paulo, Brasil \\ ${ }^{c}$ Instituto de Física Corpuscular \\ Universitat de Valencia \\ 46100 Burjassot, Valencia, Spain
}

Received 28 February, 1999

\begin{abstract}
Taking into account the stringent limits from helioseismology observations on possible matter density fluctuations described by magnetohydrodynamics theory, we find the corresponding time variations of solar neutrino survival probability due to the resonant spin-flavor precession phenomenon with amplitude of order $O(10 \%)$. We discuss the physics potential of high statistics real time experiments, like as Superkamiokande, to observe the effects of such magnetohydrodynamics fluctuations on their data. We conclude that these observations could be thought as a test of the resonant spin-flavor precession solution to the solar neutrino anomaly.
\end{abstract}

\section{Introduction}

According to all attempts to build a solution to the solar neutrino anomaly based on the resonant spinflavor precession mechanism [1], when one assumes a neutrino magnetic moment of order $\mu_{\nu}=10^{-11} \mu_{B}$, which is slightly below its present experimental limit [2], an average magnetic field of order $10^{4} \mathrm{G}[3,4]$ is required to conciliate the present solar neutrino experimental data [5]-[9] and solar neutrino theoretical predictions [10]-[15]. Using these typical values for the solar magnetic field we observed [16] that magnetohydrodynamics $[17,18]$ naturally predicts the existence of solar magnetic oscillations which period is of the order of 1 to 10 days. Resonant spin-flavor precession mechanism generates a solution to the solar neutrino anomaly only if neutrinos are very sensitive to the magnetic field as well as matter density profiles inside the Sun and, in particular, are very sensitive also to the magnetohy-

\footnotetext{
*Email:ice-fisica@acad.puccamp.br

†Email: guzzo@ifi.unicamp.br

‡Email: colonia@ifi.unicamp.br

$\S$ Email: holanda@ifi.unicamp.br
}

drodynamics fluctuations of these quantities. Therefore we expect that these fluctuations will generate an accordingly fluctuating experimental neutrino counting rate. The corresponding observable signal of this phenomenon is a periodical time fluctuation of the solar neutrino flux, in particular, in the high energy portion of the solar neutrino spectrum, detected in present real time experiments $[9,19]$. These effects can be thought as a test to the resonant spin-flavor precession solution to the solar neutrino problem since they can clearly be distinguished from other sources of time modulation in the solar neutrino observations like the effect of seasonal variation of the Sun-Earth distance on vacuum oscillations, MSW effects at the Earth, and solar magnetic activity related to the appearance of sunspots on the solar surface.

In general, magnetohydrodynamics $[17,18]$ predicts two different kinds of stable fluctuations. Alfvén waves, when transverse oscillations of the fluid with respect 
to the magnetic field and the propagation vector are present and, therefore, no fluctuation of matter density or pressure is observed. And magnetosonic waves, which present longitudinal oscillations of density with respect to the propagation vector. In reference [16] we analyzed the effects of purely Alfvén waves where only magnetic fluctuations affects the solar neutrino survival probability. Nevertheless, if matter density perturbations are present in the way described by magnetohydrodynamics, their effects can be important for the relevant high energy neutrinos observed in real time experiments [9, 19]. There are two main reasons which can be evoked to justify this. The presence of matter suppresses, in general, the neutrino spin-flavor precession mechanism, once that matter appears in the diagonal entries of the evolution matrix, which are spinflavor conserving. That is why the presence of a resonance, a position along the neutrino trajectory where the matter effects are cancelled by a term proportional to the squared mass difference divided by the neutrino energy, is important for the neutrino spin-flavor conversion. Therefore, fluctuations of the matter density can generate modifications in this resonance cancellation leading to important variations on the final probability of neutrino spin-flavor precession. The second reason to consider the effect of density fluctuations is the peculiar behavior of the adiabaticity parameter in the resonant spin-flavor precession phenomenon. In fact, several authors discussed the effect of density variations on the solar neutrino matter enhanced transitions, the MSW effect [20]. In this context, they conclude that the effects of density perturbations on transitions of solar neutrinos may be substantial if these perturbations occur in the central region of the Sun and therefore only low energy neutrinos are sensitive to such effects since their resonance layer occurs deeper in the Sun.
Note however that this conclusion cannot be straightforward invoked here. While the adiabaticity increases with increasing resonance density for the MSW effect, it is inversely proportional to the resonance density for the spin-flavor precession phenomenon and the effects of density perturbations in the solar convective zone may be important. This low density region is relevant for high energy solar neutrinos since a resonance for such neutrinos can be found there. Consequently high neutrinos observed in real time experiments can be sensitive to matter fluctuations in this region.

In this paper we investigate the impact of matter density fluctuations induced by sonic magnetohydrodynamics waves on observable time fluctuations of high energy solar neutrino flux detected in real time experiments and compare these effects with consequences from purely magnetic (Alfvén) fluctuations. We conclude that both magnetic and density fluctuations can be equally relevant, although matter density fluctuations are much more constrained from helioseismology observations. Taking into consideration such constraints, we still expect to find periodical time fluctuations in solar neutrino counting rate due to magnetohydrodynamics matter density waves of order of $10 \%$ of the total number of events.

\section{Magnetohydrodynamics and neutrino spin-flavor preces- sion}

We are assuming a non-vanishing neutrino transition magnetic moment. In this case, the interaction of neutrinos with a magnetic field will generate spin-flavor precession which is given by the evolution equations [21]

$$
i \frac{d}{d r}\left(\begin{array}{c}
\nu_{R} \\
\nu_{L}
\end{array}\right)=\left(\begin{array}{cc}
\frac{\sqrt{2}}{2} G_{F} \alpha N_{e}(r)-\frac{\Delta}{4 E} & \mu_{\nu}\left|\vec{B}_{\perp}(r)\right| \\
\mu_{\nu}\left|\vec{B}_{\perp}(r)\right| & -\frac{\sqrt{2}}{2} G_{F} \alpha N_{e}(r)+\frac{\Delta}{4 E}
\end{array}\right)\left(\begin{array}{c}
\nu_{R} \\
\nu_{L}
\end{array}\right)
$$

where $\nu_{L}\left(\nu_{R}\right)$ is the left (right) handed component of the neutrino field, $\Delta=\left|m_{L}^{2}-m_{R}^{2}\right|$ is their squared mass difference, $E$ is the neutrino energy, $G_{F}$ is the Fermi constant, $N_{e}(r)$ is the electron number density distribution and $\left|\vec{B}_{\perp}(r)\right|$ is the transverse component of the magnetic field. Finally, we are assuming that the neutron number density is $\sim 1 / 6$ of the electron number density in any point in the Sun, which gives us an $\alpha=5 / 6$ for Majorana neutrinos, in which case the final right-handed states $\nu_{R}$ are active nonelectron antineutrinos. For Dirac neutrinos, $\alpha=11 / 12$, in which case the right-handed final states are sterile nonelectron 
neutrinos [21]. In this paper we will assume Majorana neutrinos. Note however that the multiplicative factor $11 / 10$ which has to be included in $\alpha$ for Dirac neutrinos does not lead to important alterations in our conclusions, which are, in this way, valid for Majorana or Dirac neutrinos.

From Eq. (1) we observe that the neutrino spinflavor precession mechanism depends on both the magnetic field $\left|\vec{B}_{\perp}(r)\right|$ as well as the matter density distribution $N_{e}(r)$ along the solar neutrino trajectory. These quantities are affected by magnetohydrodynamics fluctuations. In order to describe them, we consider the linearized magnetohydrodynamics equations [17, 18], following the same steps of reference [16], where magnetohydrodynamics spectrum is generated by small displacements $\vec{\xi}$ from an equilibrium configuration. We are particularly interested in the magnetic and matter density fluctuations generated by the displacement $\vec{\xi}$ given, respectively, by [22]

$$
\delta \vec{B}=\vec{\nabla} \times\left(\vec{\xi} \times \vec{B}_{0}\right) \quad \text { and } \quad \delta \rho=\vec{\nabla} \cdot(\rho \vec{\xi}) .
$$

where $\vec{B}_{0}$ is the equilibrium magnetic field configuration assumed to be the one relevant to the solar neutrino problem described in reference [3] and $\rho$ is the solar matter distribution calculated in the standard solar model [10]-[14].

A crucial region of the magnetohydrodynamical spectrum is the continuum region which is associated with singularities of the Hain-Lüst equation [16, 22]. This happens when the frequency of the magnetohydrodynamical fluctuation is equal to

$$
w_{A}^{2}=\frac{k^{2} B_{0}^{2}}{\rho} \quad \text { or } \quad w_{S}^{2}=\frac{\gamma p}{\gamma p+B_{0}^{2}} \frac{k^{2} B_{0}^{2}}{\rho},
$$

where $p$ is the pressure and $\gamma=C_{p} / C_{v}$ is the ratio of specific heats. $w^{2}=w_{A}^{2}$ and $w^{2}=w_{S}$ define the Alfvén and slow continuum regions in the magnetohydrodynamical spectrum [22]. Magnetic waves and matter density fluctuations associated with these frequencies are called localized modes since they present the interesting feature of been highly peaked around the position, $r_{s}$, where the singularity occurs [23].

In order to numerically overcome the singularities associated with the continuum spectra, a resistivity layer in the position of the singularity is introduced [24]. The width of this layer is directly related with the width $\delta r$ of the localized magnetic or matter density fluctuation which can be estimated [25]:

$$
\delta r \approx 8 \pi\left(\frac{\eta}{\mu_{0} \omega\left(r_{s}\right)}\right)^{1 / 3}\left(\frac{2 B^{\prime}}{B}-\frac{\rho^{\prime}}{\rho}\right)^{-1 / 3}
$$

where $\mu_{0}$ is the vacuum permeability. In our particular scenario, $\delta r$ can achieve $10^{-1}$ (normalized by the solar radius).
To analyze the consequences of the localized waves on the solar neutrino observations, we have now to define the amplitude of possible magnetic and matter density fluctuations. The solar magnetic field is relatively free to fluctuate since the magnetic pressure $B^{2} / 8 \pi$ is negligibly small when compared with the dominant gas pressure $p[10]$ if we consider the matter density distribution $\rho$ predicted by the standard solar model and the magnetic field strength of order of those ones required to solve the solar neutrino anomaly [3]. In fact, in this case, $B^{2} / 8 \pi p$ varies from approximately $10^{-6}$ in the central regions of the Sun to order $10^{-4}$ close to the solar surface. From this argument, the magnetic field can be as large as $10^{9} \mathrm{G}$ in the solar core or $10^{7}$ $\mathrm{G}$ in the solar convective zone. More stringent bounds on the magnetic field in the convective zone are found in refs. [26, 27] where the discussion is based on the non-linear effects which eventually prevents the growth of magnetic fields created by the dynamo process. By equating the magnetic tension to the energy excess of a sinking element at the bottom of the convective zone, Schmitt and Rosner [26] obtained few times $10^{4} \mathrm{G}$ as an upper bound. Therefore fluctuations of the solar magnetic field of the same order of magnitude of the magnetic field used in reference [3] can be found inside the Sun. Despite this fact, they cannot be arbitrarily large when we are considering the linearized magnetohydrodynamics equations $[16,22]$. This implies that the displacements $\vec{\xi}$ must be small, $|\vec{\xi}|<1$, such that the non-linear terms can be neglected. This implies that $|\delta \vec{B}| /\left|\vec{B}_{0}\right|<1$. The error associated with this approximation is $\sigma \approx\left(|\delta \vec{B}| /\left|\vec{B}_{0}\right|\right)^{2}$. The maximum possible value for the ratio $|\delta \vec{B}| /\left|\vec{B}_{0}\right|$ is related with a clear statistical distinction between the maximum and the minimum value of the perturbed magnetic field, which is given approximately by $\left(|\delta \vec{B}| /\left|\vec{B}_{0}\right|\right) / \sigma$ (in units of $\sigma$ ). To have a minimum $2-\sigma$ distinction between the maximum and minimum magnetic field, we must have a maximum value of the perturbation $\approx|\delta \vec{B}| /\left|\vec{B}_{0}\right|=0.5$.

Something very different happens with possible matter density fluctuations. In fact, they are very constrained by helioseismology observations. The largest density fluctuations $\delta \rho$ inside the Sun are induced by temperature fluctuations $\delta T$ due to convection of matter between layers with different local temperatures. An estimate of such effect is presented in reference [28] and gives

$$
\frac{\delta \rho}{\rho}=m_{p} g\left(r-r_{0}\right) \frac{\delta T}{T^{2}}=\frac{r-r_{0}}{R_{0}} \frac{\delta T}{T}
$$

where $m_{p}$ is the nucleon mass, $g(r)$ is the gravity acceleration and $R_{0} \approx 0.09 \times R_{\odot}\left(R_{\odot}\right.$ is the solar radius $)$ is a numerical factor coming from the approximately exponentially decreasing standard matter density distribution inside the Sun [10]. Since $\sqrt{\left\langle\delta T^{2}\right\rangle} / T \approx 0.05$ 
is not in conflict with helioseismology observations [29], taking $\left(r-r_{0}\right) / R_{0} \approx 1$, we have to consider density fluctuations $\delta \rho / \rho$ smaller than $10 \%$. In fact, in an accurate analysis of helioseismology consequences on matter density fluctuations [30] it was concluded $\delta \rho / \rho$ can be very large (larger than $10 \%$ ) only for very inner parts of the Sun $(r<0.04)$ as well as for very superficial regions $(r>0.98)$. For $0.04<r<0.25, \delta \rho / \rho$ decreases approximately linearly and achieves its smaller value $2 \%$ in $r \approx 0.25$. Finally in the region where $0.4<r<0.9$, $\delta \rho / \rho$ is approximately $5 \%$. We impose these constraints as boundary conditions for the amplitudes of density fluctuations we will consider in the following.

Finally we can analyze the consequences of the localized magnetohydrodynamics modes on the solar neutrino flux solving the neutrino evolution equations when a non-vanishing neutrino transition magnetic moment $\mu_{\nu}$ is assumed. We will adopt here a phenomenological approach, in close analogy to what was done in reference [16]. We will assume that magnetohydrodynamics introduces gaussian shaped fluctuations which will be added to the equilibrium configuration of both matter density and/or magnetic field profile. This gaussian perturbation is centered in $r_{s}$, with width $\delta r$. For the matter density fluctuation we have:

$$
\delta \rho(r, t)=\epsilon_{\rho} \rho\left(r_{s}\right) \exp \left[-\left(\frac{r-r_{s}}{\delta r}\right)^{2}\right] \sin \left[w\left(r_{s}\right) t\right],
$$

where $\epsilon_{\rho}$ is the fluctuation amplitude normalized to the value of the standard matter density $\rho(r)$ calculated in the position of the singularity $r_{s}$ and $\delta r$ is the width of the fluctuation. The frequencies $w\left(r_{s}\right)=w_{A}$ or $w\left(r_{s}\right)=w_{S}$ are given in equation (3) and introduce a periodical time modulation on the standard matter density profile. Similarly, our assumption for the magnetic fluctuations is obtained from the above equation substituting $\rho \rightarrow B_{0}$, i.e., the standard matter density by the equilibrium field profile. Therefore the final matter density (as well as the magnetic field profile) will be given by some equilibrium profile summed to the perturbation shown in Eq. (6). We will assume the parameter $\epsilon_{\rho}$ and $\epsilon_{B}$ varying from 0 to 0.05 , which means that the matter density as well as the magnetic field fluctuate around their equilibrium values with maximum amplitude around $5 \%$ of this value. These fluctuations will generate an according time fluctuation of the neutrino counting governed by the evolution equations (1).

Since we have a very stringent experimental limit on the neutrino magnetic moment [2], a large magnetic field is necessary to find a relevant spin-flavor conversion of neutrinos which propagating through it. In this paper we will consider a magnetic field profile proposed in reference [3] which is as large as $10^{6}$ to $10^{7} G$ in the central regions of the Sun and fall by two orders of magnitude when the convective zone is reached [31]:

$$
B_{0}(r)= \begin{cases}a_{1}\left(\frac{0.2}{r+0.2}\right)^{2} \mathrm{G} & \text { for } \quad 0<r \leq 0.7 \\ B_{C} & \text { for } \quad r>0.7\end{cases}
$$

where $B_{C}$ is the magnetic field in the convective zone given by the following profiles:

$$
B_{C}=a_{2}\left[1-\left(\frac{r-0.7}{0.3}\right)^{n}\right] \mathrm{G} \quad \text { for } 0.7<r \leq 1.0
$$

or

$B_{C}=a_{2}\left[1+\exp \left(\frac{r-0.95}{0.01}\right)\right]^{-1} \mathrm{G}$ for $0.7<r \leq 1.0$.

$a_{1} \approx 10^{5}-10^{7}$ and $a_{2} \approx 10^{4}-10^{5}$ in such a way that the continuity of the magnetic field at the point $r=0.7$ is satisfied and $n=2,6$ and 8 . We assume also that the magnetic equilibrium profile $B_{0}(r)$ is in the $z$ direction. For the solar scenario, $\gamma p>>B_{0}^{2}$ and therefore $w_{A} \approx w_{S}$.

In Fig. 1 we show the periods of the magnetic and matter density fluctuations associated with the continuum spectra given in (3) for several magnetic field profiles given by the Eqs. (7)-(9). We observe that for the considered magnetic fields typical periods vary from $O(1)$ to $O(10)$ days. Time fluctuations of solar neutrino observations presenting periods of this order of magnitude constitute the most important signal of the existence of solar magnetohydrodynamics fluctuations and a crucial test for the resonant spin-flavor neutrino precession mechanism to the solar neutrino problem [35].

\section{Results}

The fluctuations of the magnetic and matter density induce fluctuations in the survival probability $P\left(\nu_{L} \rightarrow\right.$ $\left.\nu_{L}\right)$. We assume that this probability doesn't depend on the neutrino production point, and no significant oscillation occurs on the neutrino way from the Sun surface to the detector, at Earth. We also assume that no Earth effect will be present. So, the survival probability is obtained simply by integrating numerically Eq (1), from the neutrino production point to the solar surface, with the inicial condition that all neutrinos are produced as left-handed neutrinos, $\left(\nu_{R}, \nu_{L}\right)_{t=0}^{T}=(0,1)^{T}$. The integration gives us the neutrino state at the solar surface, and then the survival probability is calculated using $P\left(\nu_{L} \rightarrow \nu_{L}\right)=\left|\left\langle\nu_{L} \mid \nu(t)\right\rangle\right|^{2}$. We have done this integration with a subroutine that uses a Runge Kutta technique of fourth order. 


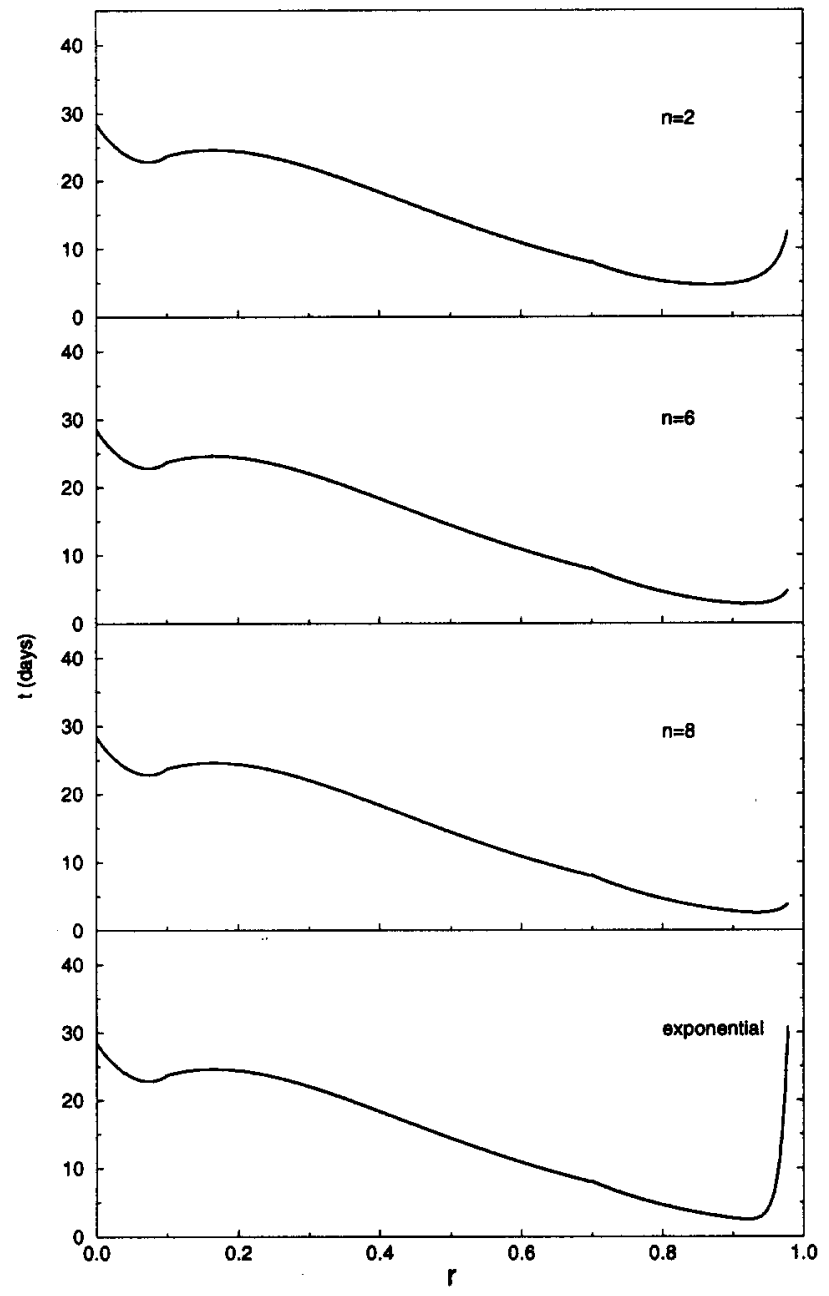

Figure.1 Periods of the magnetohydrodynamics fluctuations for the continuum spectra when the magnetic profile shown in Eq. (8) when $n=2, n=6, n=8$ and exponential profile of Eq. (9). We adopted the parameters $a_{1} \approx 1.0 \times 10^{6} \mathrm{G}$, $a_{2} \approx 4.0 \times 10^{4} \mathrm{G}$ and $k \approx 10^{-10} \mathrm{~cm}^{-1} .0$.

The results of our analysis are shown in Fig. 2. In this figure we present the amplitude $\Delta P$ of the fluctuations of the survival probability as a function of $\Delta m / 4 E$, for some values of $r_{S}$, the position of the localized mode. This fluctuations are calculated computing the survival probability at distincts moments $t$ of the perturbations in Eq. (6), and selecting the moments when this probability reaches a maximum and a minimum. The amplitude of the fluctuations is the difference between these two values. We show the phenomenon for $r_{S}=0.5,0.9$ and for the value of $r_{S}$ which gives the maximum probability amplitude, usually around $r_{S} \approx 0.7$. We adopted the magnetic field profile given in Eqs. (7) and (8), with $n=6$ and $\mu_{\nu}=1 \times 10^{-11} \mu_{B}$. Other magnetic field profiles shown in Eqs. (7), (8) and (9) generate very similar consequences of magnetohydrodynamics fluctuations on the solar neutrino survival probability and we will not show them here. We assumed $\epsilon_{\rho}$ and $\epsilon_{B}$ in Eq. (6), i.e., the relative amplitude of matter density and magnetic field profile fluctuations, respectively, to vary from 0 to $5 \%$ as it is indicated in Fig. 2. Also, vertical lines indicate the value of $\log (\Delta m / 4 E)$ corresponding to a resonance coinciding with the indicated singularity $r_{S}$. We can observe that the fluctuations of the survival probability are maxima near the regions of the resonances and are well localized in $\Delta m / 4 E$ for $r_{s} \leq 0.7$.

We verified also that, for fixed values of $r_{S}$ and $\Delta m / 4 E$, the corresponding probability amplitude varies linearly with the magnitude of the amplitude of the matter density $\delta \rho$ and/or magnetic field fluctuation $\delta \vec{B}$. For instance, when $\epsilon_{\rho}=\epsilon_{b}$, the maximal probability fluctuation corresponding to the magnetic fields given in Eq. (8) occurs in $r_{S}=0.69$ and can be written as $\Delta P \approx 2.20 \times 10^{-2} \epsilon_{\rho}$ for $n=2, \Delta P \approx 2.48 \times 10^{-2} \epsilon_{\rho}$ for $n=6$ and $\Delta P \approx 2.64 \times 10^{-2} \epsilon_{\rho}$ for $n=8$. Considering the exponential behavior for the magnetic field in the convective zone, the maximal amplitude of survival probability occurs in $r_{S}=0.68$ and presents the following linear behavior with $\epsilon_{\rho}: \Delta P \approx 2.81 \times 10^{-2} \epsilon_{\rho}$. Therefore, given the results of Fig. 2, one can easily infer the amplitude of the survival probability for other values of $\delta \rho$ and $\delta \vec{B}$. Note that this approximately linear behavior is valid for $\epsilon_{\rho}<0.2$, which includes the physical limit for helioseismologically acceptable variations of the matter density.

From Fig. 2, we observe also that the maximal effect of the matter density and/or magnetic field fluctuation on the survival probability occurs if these magnetohydrodynamics fluctuations are found in the beginning of the convective zone where $r_{S} \approx 0.7$. This can be understood when we examine the behavior of the adiabaticity parameter for the spin-flavor conversion phenomenon. As we have already mentioned, this parameter increases with $r$ and approaches the unit for $r \approx 0.7$. In fact, it was pointed out previously (see, for instance [36]) that this is a privileged situation for the neutrino conversion. Note also that according to references $[3,4]$, the required value of $\Delta m$ to find a solution to the solar neutrino anomaly is $O\left(10^{-7}\right) \mathrm{eV}^{2}$ or smaller. If $\Delta m=O\left(10^{-7}\right) \mathrm{eV}^{2}$, neutrinos of $O(10) \mathrm{MeV}$ in energy will experience their resonance around $r=0.7$ and therefore will be very sensitive to the maximal amplitude probability indicated in Fig. 2. This is exactly the energy range probed by real time experiments, like as Superkamiokande [9] and SNO [19], which are, therefore, very suitable to investigate the hypothesis of solar magnetohydrodynamics perturbations and their effects on solar neutrinos. 


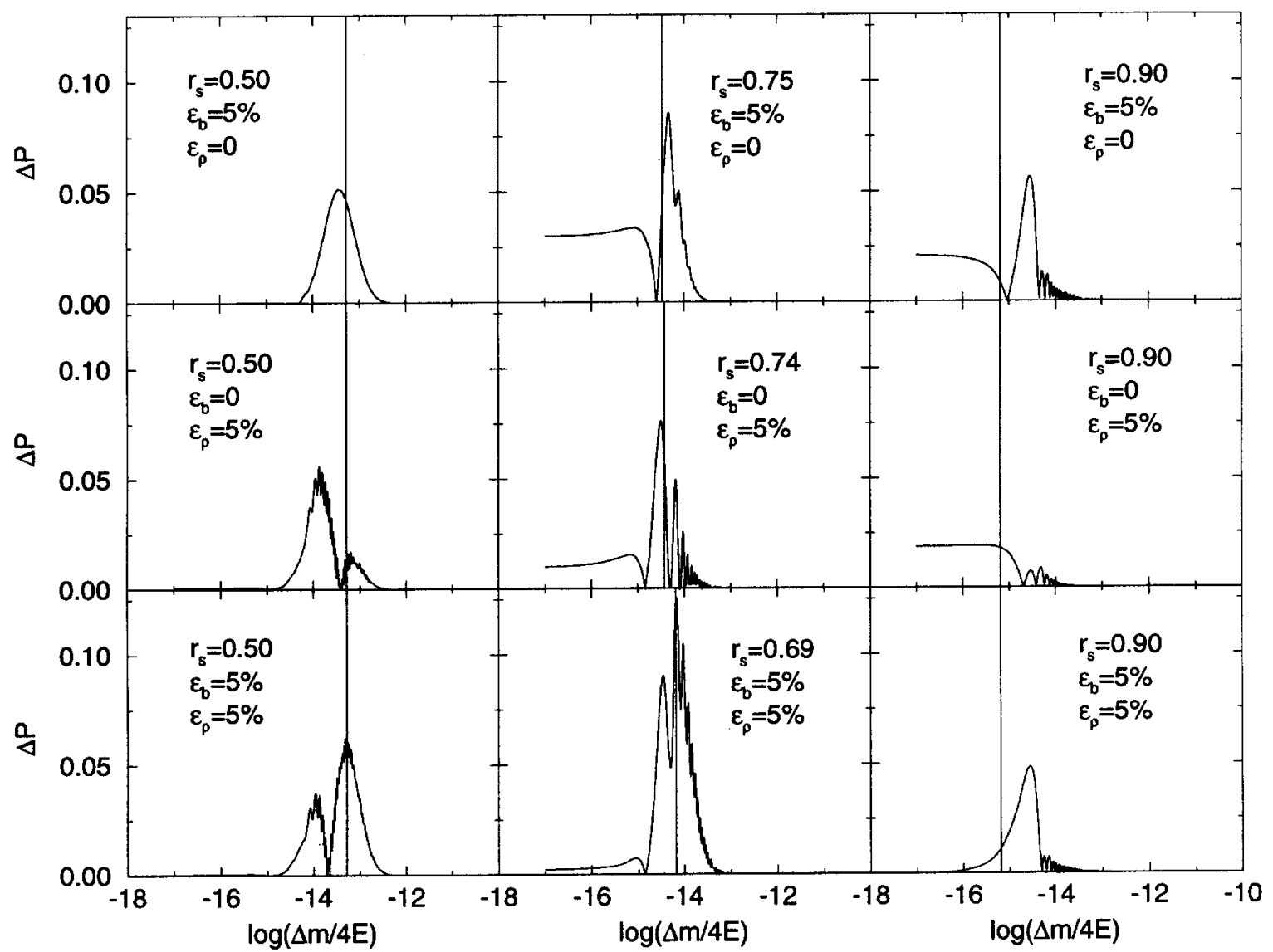

Figure.2 Amplitude $\Delta \mathrm{P}$ of the survival probability in function of $\Delta m / 4 E$ for some values of $r_{s}$. The maximum probability amplitude occurs around $r_{s}=0.7$. The vertical lines correspond to a resonance coinciding with the singularity $r_{s}$.

After several hundred days of taking data, Superkamiokande observations are, in principle, able to verify the existence of time fluctuation with period from $O(1)$ to $O(10)$ days. Unfortunately, these data are not published nor available. Nevertheless we can argue the potential of this experiment to perform such analysis. Fig. 3 shows the amplitude of the expected event rates in Superkamiokande experiment when we assume that a magnetohydrodynamics fluctuation is localized around $r_{S} \approx 0.7$. We convulated the standard ${ }^{8} B$-neutrino production spectrum [10], the $\nu_{e}-e^{-}$scattering crosssection increasing linearly with energy and the experimental efficiency which is approximately $20 \%$ for neutrino energies around $5 \mathrm{MeV}$ up to a maximum of $70 \%$ for neutrino energies of $10 \mathrm{MeV}$ or larger. These event rates are given in solar neutrino units (SNU) for several values of $\Delta m$. We observe that for $\Delta m=3 \times 10^{-7} \mathrm{eV}^{2}$, the maximum event rate amplitude is obtained. I.e., after having been precessed due to the existence of a sonic magnetohydrodynamical waves, the number solar neutrinos scattering with Superkamiokande electrons fluctuates with amplitude around 0.003-0.004 SNU, if these neutrinos present energies around 8 and $10 \mathrm{MeV}$. This is approximately $10-15 \%$ of the total rate of scattering events in this energy range. Note that if $\Delta m$ is different from the values shown in Fig. 3, this means that neutrinos with energies around $5-15 \mathrm{MeV}$ will experience resonance in a position different from $r_{s} \approx 0.7$ and, although not maximal, their counting rate will still fluctuate.

\section{Conclusions}

Magnetohydrodynamics predicts magnetic as well as matter density fluctuations in the Sun. The purpose of this paper is to analyze the effects of possible solar matter density fluctuations on solar neutrino observations taking into account the stringent limits on these fluctuations coming from helioseismology. We verify also the potential of high statistics real time solar neutrino experiments, like as Superkamiokande, to observe these effects. We showed that the survival probability fluctuations of active solar neutrinos due to the resonant neutrino spin-flavor precession can be of order $10 \%$ if amplitudes of the magnetic or matter density fluctuations $\epsilon_{b}$ or $\epsilon_{\rho}$ are of order of $5 \%$ (20\% if $\epsilon_{b}=\epsilon_{\rho}=10 \%$ ). Therefore, in order to make evident the existence of these magnetohydrodynamics fluctuations in the Sun, 


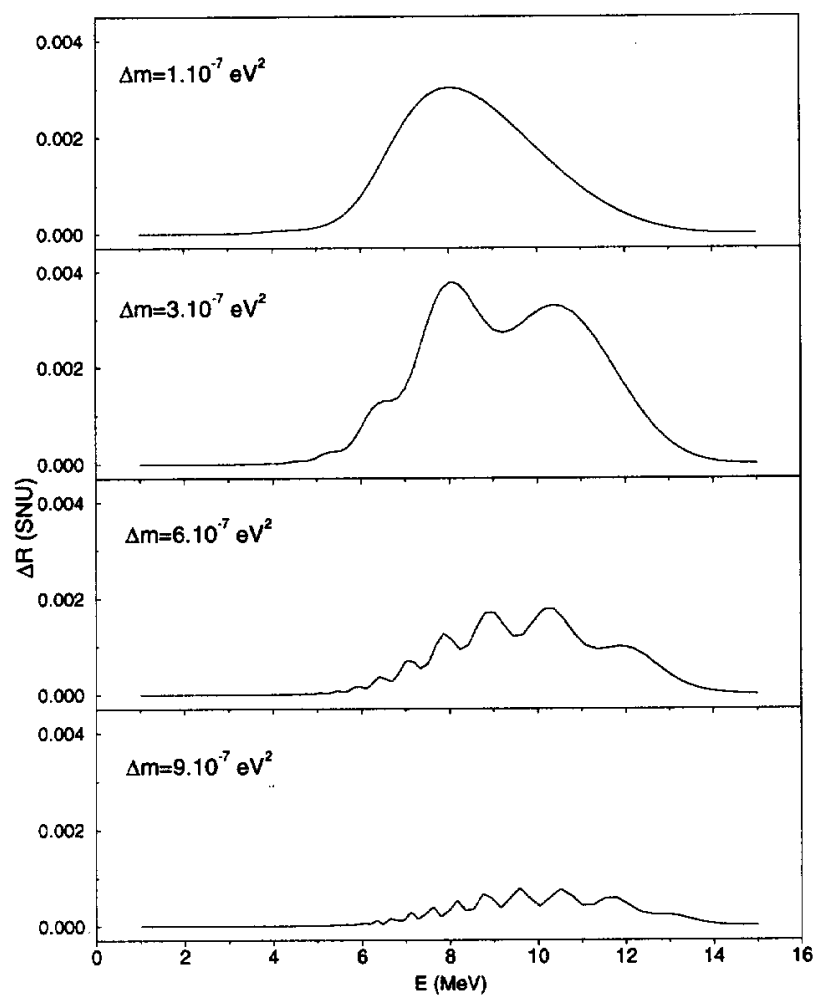

Figure.3 Amplitude $\Delta \mathrm{R}$ of the expected event rates to Superkamiokande experiment for some values of the mass square difference $\Delta m$

we have to look for solar neutrino data time fluctuations of this order in the time scale compatible with the periods predicted by the theory. From Figure 1, we see that relevant time scale can be of order $O(1)$ to $O(10)$ days. Collecting $O(20)$ events a day, Superkamiokande presents a statistics error of approximately $10 \%$ in an interval of 5 days. Furthermore, a Fourier analysis of the experimental observations fitting a period of time of the order of the total period of Superkamiokande observation (of order of hundred days), could drastically reduce the involved errors and the amplitudes of order of $10 \%$ shown in Figures 1 and 2 could be experimentally tested. There is no available published solar neutrino data to conduct a conclusive analysis of possible data fluctuation with period $O(1-10)$ days. Analysis of time fluctuations on solar neutrino data conducted up to now, privileged different periods: exact 24 hours as a result of MSW effect inside the Earth, six months to conciliate seasonal variation and neutrino vacuum oscillations or 11 years to verify solar magnetic activity effects on spin flip phenomenon.

We conclude that Superkamiokande is potentially interesting to investigating the effects on solar neutrino observations coming from magnetohydrodynamics fluctuations. The observation of these effects could be taken as an evidence of the resonant spin-flavor precession mechanism in the Sun.

\section{Acknowledgements}

This work was supported by Fundação de Amparo à Pesquisa do Estado de São Paulo (FAPESP), Programa de Apoio a Núcleos de Excelência (PRONEX) and Conselho Nacional de Desenvolvimento Científico e Tecnológico (CNPq).

\section{References}

[1] A. Cisneros, Astrophys. Space Sci. 10 (1970) 87; M. Voloshin, M.I. Vysotsky and L. Okun, Sov. J. Nucl. Phys. 44 (1986) 440; J. Schechter and J.W.F. Valle, Phys. Rev. D 24 (1981) 1883; E. Akhmedov, Sov. J. Nucl. Phys. 48 (1988) 382; Phys. Lett. B213 (1988) 64; C.S. Lim and W.J. Marciano, Phys. Rev. D37 (1988) 1368.

[2] C. Caso et al., "Review of Particle Physics", The European Physical Journal C3 (1998) 1.

[3] E.Kh. Akhmedov, A. Lanza and S.T. Petcov, Phys. Lett. B 303 (1993) 85.

[4] M.M. Guzzo and H. Nunokawa, "Current status of the Resonant Spin-flavor Solution to the solar neutrino problem", HEP-PH/9810408.

[5] K. Lande (Homestake Collaboration) in Neutrino '98, Proceedings of the XVIII International Conference on Neutrino Physics and Astrophysics, Takayama, Japan, 4-9 June 1998, edited by Y. Suzuki and Y. Totsuka, to be published in Nucl. Phys. B (Proc. Suppl.), scanned transparencies are available at URL http://wwwsk.icrr.u-tokyo.ac.jp/nu98/scan/index.html.

[6] Y. Fukuda et al. (Kamiokande Collaboration), Phys. Rev. Lett. 77 (1996) 1683.

[7] V. Gavrin (SAGE Collaboration) in Neutrino '98 [5].

[8] T. Kirsten (GALLEX Collaboration) in Neutrino '98 [5].

[9] Y. Suzuki (SuperKamiokande Collaboration) in Neutrino '98 [5].

[10] J. Bahcall and R. Ulrich, Rev. of Mod. Phys. 60, 297 (1988); J. N. Bahcall, Neutrino Astrophysics, Cambridge University Press, New York, (1989).

[11] J. N. Bahcall and M.H. Pinsonneault, Rev. of Mod. Phys. 67 (1995) 781.

[12] J. N. Bahcall, S. Basu and M.H. Pinsonneault, Phys. Lett. B 433 (1998) 1.

[13] For a recent review, see J. N. Bahcall, astro$\mathrm{ph} / 9808162$.

[14] E. G. Adelberger et al., astro-ph/9805121, Rev. Mod. Phys., (to be published, October 1998).

[15] J. N. Bahcall, P. I. Krastev and A. Yu. Smirnov, Phys. Rev. D58 (1998) 096016.

[16] M.M. Guzzo, N. Reggiani and J.H. Colonia, Phys. Rev. D56 (1997) 588.

[17] E.R. Priest, Solar Magnetohydrodynamics, D.Reidel Publishing Company (1987). 
[18] P. Foukal, Solar Astrophysics, Wiley-Interscience Publication (1989).

[19] G.T. Ewan, "Sudbury Neutrino Observatory", in Frontiers of Neutrino Astrophysics, edited by Y. Suzuki and K. Nakamura, Universal Academy Press, Tokyo (1993) p. 135 .

[20] P.I. Krastev and A. Yu. Smirnov, Phys. Lett. B 226 (1989) 341; Mod. Phys. Lett. A 6 (1991) 1001; A. Abada and S.T. Petcov, Phys. Lett B 279 (1992) 153; F.N. Loretti and A.B. Balantekin, Phys. Rev. D 50 (1994) 4762.

[21] For a review on neutrino spin-flavor precession see J. Pulido, Phys. Rep. 211 (1992) 167.

[22] J.P Goedbloed and P.H. Sakanaka, The Physics of Fluids 17 (1974) 908.

[23] J.P. Goedbloed, Physica 12D (1984) 107.

[24] L. Villard. K. Appert, R. Grubber and J. Vaclavik, Comp. Phys. Rep. 4 (1986) 95.

[25] J. M. Kappraf and J. A. Tataronis, J. Plasma Physics 18 (1977) 209; T. Sakurai, M. Goossens and J. V. Hollweg, Solar Physics 133 (1991) 227.

[26] J. Schmitt and R. Rosner, Astrophys. J. 265 (1983) 901.
[27] X. Shi et al., Comm. Nucl. Part. Phys. 21 (1993) 151.

[28] H. Nunokawa, A. Rossi, V.B. Semikoz and J.W.F. Valle, Nuc. Phys. B472 (1996) 495.

[29] S. Turck-Chiéze et al., Phys. Rep. 230 (1993) 57.

[30] G. Fiorentini and B. Ricci, "Solar neutrinos: where we are and what is next?" ASTRO-PH/9801185.

[31] Some mechanisms to generate such fields are suggested in A. Cisneros in rerefence [1] and D. Moss, Monthly Notes R. Astron. Soc. 224 (1987) 1019.

[32] E. Parker, Astrophys. J. 198 (1975) 205.

[33] V.D. Kuznetsov and S.I. Syrovastskii, Sov. Astron. 23 (1979) 715; V.N. Krivodubskii, Sov. Astron. Lett. 13 (1987) 338.

[34] J.H. Colonia, P.C. de Holanda, M.M. Guzzo and N. Reggiani, in preparation.

[35] Obviously, if fluctuations of exact one day period is observed in the solar neutrino data, MSW effect at the Earth is a natural explanation to be evoked. However any fluctuation with different from exact one day period rules out MSW.

[36] M.M. Guzzo and J. Bellandi, Phys. Lett. B317 (1993) 130. 